\title{
INFECÇÃO PELO HELICOBACTER PYLORI E SUA CORRELAÇÃO COM OS SINTOMAS DISPÉPTICOS E EVOLUÇÃO DA GRAVIDEZ
}

\author{
Sansom Henrique Bromberg*, Kioko Takei, Sidney Antonio lagrosa Garcia, André da Costa Vitor, Arnaldo Zanoto, Fausto Farah Baracat \\ Trabalho realizado no Curso de Pós-graduação em Gastroenterologia Cirúrgica do Instituto de Assistência Médica ao Servidor Público \\ (IAMSPE) e no Serviço de Obstetrícia e Ginecologia do Hospital do Servidor Público Estadual - Francisco Morato de Oliveira (HSPE-FMO)
}

\section{*Correspondência:}

Av. Angélica, $589,8^{\circ}$ andar Santa Cecília, São Paulo, SP Cep: $01227-000$

brombengsh@yahoo.com.br

\begin{abstract}
RESUMO
OBjetivo. Investigar em gestantes possível correlação da infecção pelo Helicobacter pylori com sintomas dispépticos e características maternas da gravidez.

Métodos. A soropositividade ao H. pylori foi obtida em 146 mulheres com 10 a 23 semanas de gestação. A média de idade foi de 29,6 anos, variando de 17 a 45 anos. A determinação semiquantitativa de anticorpos da classe lg $\mathrm{G}$ contra o H. pylori foi realizada com "kit" Elisa de procedência comercial (Varelisa H.pylori lgg Antibodies, Pharmacia \& Upjohn Diagnostics $\mathrm{GmbH} \&$ Co., Germany). Grávidas com sintomas dispépticos que necessitaram de tratamento constituíram o grupo investigado. Outras características maternas também foram correlacionadas à infecção pelo H. pylori: obesidade - IMC >30; paridade - primípara e multípara; evolução da gestação - a termo e aborto ou prematuridade. Os valores obtidos foram submetidos a análise estatística - t de Student e Qui-quadrado.
\end{abstract}

Resultados. A prevalência da infecção pelo H. pylori na população estudada foi de 65,7\%. Constatou-se ausência de correlação entre a infecçãa bacteriana e os fenômenos dispépticos da gravidez. Correlação negativa também foi constatada para obesidade, paridade e 0 evoluir da gestação. Gestantes com sorologia positiva apresentaram media de idade significantemente maior que as de sorologia negativa: $30,45 \pm 6,87$ versus $27,96 \pm 7,44, p=0,045565$.

Conclusão. $O$ presente estudo não associou a infecção pelo $\mathrm{H}$. pylori com os sintomas dispépticos da gravidez, nem com as características maternas estudadas: obesidade, paridade e evolução da gravidez.

UniteRmos: Helicobacter pylori. Gravidez. Dispepsia. Vômitos. Queimação retro-esternal.

\section{INTRODUÇÃO}

O Helicobacter pylori (H. pylori) é reconhecido como significante agente etiológico da gastrite em seres humanos e como fator essencial na patogenia da úlcera péptica'. Múltiplas evidências sugerem que esse microrganismo esteja também envolvido na patogênese do câncer e do linfoma MALT do estômago $0^{2,3}$.

Nos países em desenvolvimento, 70\% a 90\% da população está infectada pela bactéria; já em países industrializados a prevalência é menor, situando-se entre $25 \%$ e $50 \%$ !

A prevalência da infecção pelo $H$. pylori na dispepsia não ulcerosa mostra índices entre $30 \%$ a $70 \%$, sendo similar à da população geral $\left.\right|^{4}$.

Já a presença de sintomas dispépticos durante o primeiro trimestre da gravidez ocorre em 50\% a 90\% das mulheres grávidas e persiste, geralmente em menor escala, nos outros seis meses ${ }^{5}$.

Muitos estudos sorológicos vêm sendo empregados em escala crescente visando detectar a presença do anticorpo anti-H. pylori em gestantes, com o intuito de associar a hiperemese gravídica à positividade do teste. Os resultados destes trabalhos ainda são controversos ${ }^{5-9}$. O estudo da associação do H. pylori com sintomas dispépticos da gravidez vem merecendo menor atenção dos pesquisadores ${ }^{10,11}$.
Por outro lado, já foi sugerido ser a presença de infecção pelo H. pylori fator de risco para o desenvolvimento intra-uterino do feto ${ }^{12}$.

Em nosso meio, raras são as pesquisas sobre a prevalência do $H$. pylori em gestantes ${ }^{13}$; da mesma maneira, eventual correlação da positividade sorológica para anticorpos lgG do H. pylori com os sintomas dispépticos na gravidez e com diferentes características maternas, ainda carece de investigação.

A principal motivação deste trabalho foi a de investigar possível associação entre sintomas dispépticos da gravidez com a positividade da infecção pelo H. pylori. Pesquisou-se também eventual correlação desta infecção com a evolução da gravidez e características demográficas dessas gestantes.

\section{MÉtodos}

Foi estudada prospectivamente uma coorte de 146 mulheres com idade gestacional entre 10 e 23 semanas de gravidez atendidas no ambulatório do Serviço de Obstetrícia e Ginecologia do Hospital do Servidor Público Estadual - Francisco Morato de Oliveira. Após serem consultadas, foram encaminhadas para o Serviço de Laboratório Clínico para coleta sangüínea de exames rotineiros de pré-natal e para verificação sorológica da presença de H. pylori. Este estudo foi aprovado pelo Comitê de Ética em Pesquisa da instituição sob o protocolo 22/03. 
A idade das gestantes variou de 17 a 45 anos, com média de 29,6 anos e mediana de 31 anos. Cento e doze das mulheres eram brancas (76,7\%), 30 negras $(20,5 \%)$ e $4(2,8 \%)$ da raça amarela. Cerca de 48 (32,9\%) delas eram primíparas e 98 $(67$, I\%) multíparas.

A presença de sintomas dispépticos foi anotada durante 0 seguimento ambulatorial no primeiro e segundo trimestre gestacional. A dispepsia foi considerada positiva na persistência de três ou mais dos sintomas dor epigástrica, eructações, náuseas, peso pós-prandial, vômitos e queimação retro-esternal, que interferiam nas atividades diárias das gestantes, necessitando essas de retorno não programado ao hospital e de tratamento. Foram excluídas do estudo as grávidas com antecedentes de doenças digestivas, com doenças metabólicas ou sistêmicas, com alterações psicológicas, que receberam antibióticos ou em uso terapêutico de drogas capazes de desencadear sintomas gastrointestinais. Caracterizou-se, assim, dois grupos de gestantes: o dispéptico e o sem dispepsia, utilizado como controle.

As grávidas foram pesadas e tiveram aferidas sua altura, obtendo-se assim o índice de massa corpórea (IMC), segundo a fórmula IMC = peso / altura $x$ altura. Para efeito deste estudo, 24 (16,4\%) gestantes com pontuação acima de 30 foram consideradas obesas e I0I (69,2\%) com IMC igual ou menor que 30 consideradas de peso normal; em 21 $(14,4 \%)$ das mulheres não se conseguiu obter esse dado.

Em 123 (84,2\%) das grávidas, a evolução da gestação foi acompanhada no serviço. Cento e cinco delas (71,9\%) apresentaram gestação a termo e realizaram seus partos na instituiçãa. Das 18 $(12,3 \%)$ restantes, oito $(5,5 \%)$ tiveram parto prematuro e dez $(6,8 \%)$ abortaram. Foi considerado prematuro o recém-nascido com menos de 37 semanas.

Exame sorológico - A determinação semiquantitativa de anticorpos da classe $\lg G$ anti - Helicobacter pylori foi realizada empregando-se "kit" ELISA indireto, não competitivo, de procedência comercial (Varelisa H.pylori lgG Antibodies, Pharmacia \& Upjohn Diagnostics GmbH \& Co., Germany), de acordo com as instruções dos fabricantes. A sensibilidade deste teste é de $96 \%$ comparada a biópsias endoscópicas positivas, e sua especificidade é de $92 \%$ comparada a biópsias negativas, segundo o fabricante. Para sua realização, $100 \mathrm{ml}$ das amostras e dos soros controles diluídos a 1:101 foram adicionados às placas plásticas de microcirculação, sensibilizadas com antígenos do H. pylori acrescidos de antígeno recombinante de $120 \mathrm{hDa}$, seguido de incubação por 30 minutos a temperatura ambiente. Após quatro ciclos de lavagens das placas, 100 uL do conjugado anti-IgG humano marcado com peroxidase foi adicionado, seguindo-se novamente 30 minutos de incubação e lavagens. $\mathrm{O}$ substrato enzimático constituído de $\mathrm{H}_{2} \mathrm{O}_{2}$ e $\operatorname{TMB}\left(3,3^{\prime}, 5,5^{\prime}\right.$ tetrametilbenzidina) foi adicionado e, após 15 minutos, a reação foi bloqueada e realizada a leitura espectrofotométrica. $O$ resultado semiquantitativo, expresso em $\mathrm{U} / \mathrm{ml}$, foi obtido conforme as instruções dos fabricantes, a partir de 5 pontos do padrão, de zero a $100 \mathrm{U} / \mathrm{ml}$. Amostras com resultados superiores a $100 \mathrm{U} / \mathrm{ml}$ foram diluídas e retestadas. Os valores iguais ou acima de $10 \mathrm{U} / \mathrm{ml}$ foram considerados positivos.
Tabela I - Sorologia para $H$. pylori e sua associação com sintomas dispépticos em 146 gestantes

\begin{tabular}{|c|c|c|c|}
\hline & Grupo dispéptico & Grupo controle & Total \\
\hline H. pylori negativo & $40(36,7 \%)$ & $10(27,0 \%)$ & 50 \\
\hline H. pylori positivo & $69(63,3 \%)$ & $27(73,0 \%)$ & 96 \\
\hline $\begin{array}{l}\text { Total } \\
x^{2}=0,76 \quad p=\end{array}$ & 8400 & 37 & 146 \\
\hline
\end{tabular}

Tabela 2 - Sorologia para H. pylori e sua associação com 0 índice de massa corpórea

\begin{tabular}{|c|c|c|c|}
\hline \multirow[b]{2}{*}{ H. pylori negativo } & $\mathrm{IMC}<30$ & $I M C>30$ & Total \\
\hline & $32(31,7 \%)$ & II (45,8\%) & $\overline{43}$ \\
\hline H. pylori positivo & $69(68,3 \%)$ & $13(54,2 \%)$ & 82 \\
\hline \multicolumn{2}{|c|}{$\begin{array}{l}\text { Total } \\
X^{2}=1,15 \quad p=0,2834\end{array}$} & 24 & 125 \\
\hline
\end{tabular}

Tabela 3 - Sorologia para H. pylori e número de gestações anteriores

\begin{tabular}{|c|c|c|c|}
\hline & Multigestas & Primigestas & Total \\
\hline H. pylori negativo & $29(29,3 \%)$ & $21(44,7 \%)$ & $\overline{50}$ \\
\hline H. pylori positivo & $70(70,7 \%)$ & $26(55,3 \%)$ & 96 \\
\hline$X^{2}=1,15 \quad p=$ & 99 & 47 & 146 \\
\hline
\end{tabular}

Análise estatística - Para análise dos resultados foi adotado nível de significância de $5 \%(a=0,05)$. O teste t não pareado ou t de Student foi utilizado para comparar a idade dos grupos. Para os valores entre grupos nas tabelas $2 \times 2$ utilizou-se o teste do Qui-quadrado corrigido por Yates. Valores significantes receberam asterisco $(*)$.

\section{Resultados}

Noventa e seis gestantes apresentaram valores sorológicos iguais ou superiores a $10 \mathrm{U} / \mathrm{ml}$, com média de 65,65 U/ml (variação de $10 \mathrm{U} / \mathrm{ml}$ a $525 \mathrm{U} / \mathrm{ml})$. Assim, a prevalência de infecção pelo H. pylorinas gestantes deste estudo foi de 65,7\% (96/I46).

As grávidas com sorologia positiva apresentaram média de idade significantemente maior que as portadoras de sorologia negativa: 30,45 $\pm 6,87$ anos versus $27,96 \pm 7,44$ anos $; t=2,02$ e $p=0,045565$.

Sintomas dispépticos foram constatados em 37 (25,3\%) das gestantes (grupo dispéptico) e não se correlacionaram com a positividade sorológica para o H. pylori, como pode ser observado na Tabela I.

Tampouco houve associação de sorologia positiva com a obesidade (Tabela 2).

A soropositividade ao $H$. pylori não se correlacionou com o número de partos anteriores nem com a evolução da gestação, como pode ser observado nas Tabelas 3 e 4 . 


\begin{tabular}{lccc}
\hline \multicolumn{4}{c}{ Tabela 4 - Sorologia para H. pylori e evolução da gestação } \\
\hline & $\frac{\text { A termo }}{36(34,3 \%)}$ & $\frac{\text { Prematuro/Aborto }}{8(44,4 \%)}$ & $\frac{\text { Total }}{44}$ \\
H. pylorinegativo & $69(65,7 \%)$ & $10(55,6 \%)$ & 79 \\
H. pyloripositivo & 105 & 18 & 123 \\
$\begin{array}{l}\text { Total } \\
X^{2}=0,32\end{array} \quad p=0,5723$ & & & \\
\hline
\end{tabular}

\section{Discussão}

A detecção sorológica do $H$. pylori é procedimenta não invasivo, com alta sensibilidade e especificidade em predizer a infecção causada por essa bactéria!.

A vantagem maior no emprego dos testes sorológicos é a possibilidade de abarcar número considerável de pessoas a serem investigadas de modo rápido, simples e sensível, aliado ao seu baixo custo ${ }^{14}$. Torna-se, assim, um bom teste para ser utilizado em estudos epidemiológicos. Cuidados devem ser tomados para eventuais inespecificidades dos reagentes nas condições do laboratório executante, na detecção de variações das seroconversões ou reversões; deve-se sempre evitar a utilização do teste imediatamente após tratamento ${ }^{14}$.

Na ausência de terapêutica adequada, os níveis de anticorpos permanecem elevados por muitos anos, refletindo a permanência da infecção; baixos níveis da lgG tendem a permanecer por meses após a erradicação da bactéria?.

Tais atributos fazem deste teste método adequado na investigação em grávidas e recém-nascidos.

Possível limitação de seu emprego é representada pelo fato de serem os testes deste tipo incapazes de refletir infecção corrente ativa. Entretanto, eles são utilizados em larga escala como instrumento diagnóstico visando instituir a terapêutica contra o H. pylori .

As taxas de infecção pelo H. pylori são mais elevadas nos países em desenvolvimento. A higiene precária, mais comum em populações de menor estado socioeconômico, principalmente na infância, parece estar relacionada a alta soroprevalência da bactéria. Outras variáveis como a área geográfica, idade, etnia e nível educacional, entre outras, também influenciam sua prevalência ${ }^{15}$.

No Brasil, os estudos sobre as taxas de prevalências sorológicas do H. pylori ainda não abrangeram faixa representativa da população, visto terem sido elaborados em poucos estados do país. Rocha et al., em 1994, utilizando ELISA, relataram prevalência de 90\% em Minas Gerais $^{16}$. O mesmo grupo de pesquisadores, investigando a prevalência da bactéria na população rural de Nossa Senhora do Livramento, Mato Grosso, detectou a presença do anticorpo $\lg \mathrm{G}$ em $77,5 \%$ das crianças e em $84,7 \%$ dos adultos ${ }^{17}$.

Estudo anterior em nossa instituição, investigando a prevalência do H. pylori em pacientes com diagnóstico definitivo de doença ulcerosa gastroduodenal e em população aparentemente normal, mostrou taxas de $94,5 \%$ e $37 \%$, respectivamente ${ }^{13}$.

Quando comparados a assintomáticos, pacientes dispépticos parecem apresentar prevalência maior de sorologia positiva.
Estudo realizado no Kenya encontrou soropositividade em $71 \%$ dos dispépticos e em $51 \%$ dos assintomáticos ${ }^{18}$.

A soroprevalência em grávidas assintomáticas também parece variar de acordo com a área geográfica. $\mathrm{Na}$ Europa, a prevalência variou de $10,6 \%$ a $21,3 \% 19,20$.

Estudo belga envolvendo 542 mulheres em seu seguimento pré-natal mostrou positividade sorológica para $\circ \mathrm{H}$. pylori em I $20(22,1 \%)$ delas $^{21}$.

Em populações de gestantes asiáticas - Turquia e Formosa - e africanas - Egito, esses valores se mostraram muito superiores: $44,8 \%$, $62,5 \%$ e $88 \%$, respectivamente $9,22,23$.

A prevalência de sorologia positiva nas 146 gestantes do presente estudo foi de $65,7 \%$ (96/I 46), nos níveis desses últimos países.

Esses valores são muito superiores aos $37 \%$ encontrados em indivíduos aparentemente normais nesta mesma instituição' ${ }^{13}$.

Vários pesquisadores constataram aumento significante da soropositividade com a idade ${ }^{10}$. No estudo belga acima citado, a faixa etária entre 20 e 30 anos mostrou positividade de 16,5\% enquanto a de 30 a 40 anos foi de $27,2 \%{ }^{21}$.

Os valores significantes obtidos neste trabalho, 30,45 \pm 6,87 anos para as mulheres soropositivas e 27,96 \pm 7,44 para as soronegativas corroboraram os da literatura. A maior facilidade de entrar em contato com pessoas soropositivas poderia explicar essa prevalência sorológica do H. pylori nas faixas de mais idade.

Embora os sintomas dispépticos estejam presentes em número considerável de grávidas, pouco se conhece a respeito de sua etiologia.

O refluxo esofágico estaria relacionado à pressão mecânica exercida pelo útero aumentado e ao tônus diminuído do esfíncter inferior do esôfago induzido por hormônios, principalmente pela progesterona ${ }^{24}$.

$\mathrm{Na}$ gestação em início, verifica-se acúmulo de fluidos e o deslocamento de volume do intra para o extracelular em virtude do aumento de esteróides hormonais, resultando em alteração do $\mathrm{pH}$. No território digestivo, este fato poderia desencadear atividade de populações de $H$. pylori até então latente, propiciando ou incrementando o aparecimento de sintomas dispépticos ${ }^{6}$.

Hormônios gestacionais seriam os agentes mais envolvidos por propiciar alterações da motilidade gastrintestinal e induzir ao relaxamento da musculatura parietal. Daí a explicação para o mau esvaziamento gástrico, tempo prolongado de trânsito intestinal e fenômenos espásticos ${ }^{24}$.

Por outro lado, alguns autores sugeriram que a presença de fenômenos dispépticos ajudaria a proteger o feto de eventuais produtos nocivos da dieta consumida, e consideraram esses sintomas como fisiológicos ${ }^{25}$.

Os resultados de trabalhos sobre a associação de fenômenos dispépticos em grávidas com $\mathrm{H}$. pylori são controversos, embora a maioria deles não constate tal correlação.

Em Porto Rico, a pesquisa sorológica mostrou-se positiva em 89\% de 45 grávidas com acentuada dispepsia matinal e somente em $7 \%$ de 44 grávidas sem esses sintomas ${ }^{26}$. Na Irlanda do Norte, a incidência de 
fenômenos dispépticos gestacionais foi a mesma em grávidas H.pylori positivas e negativas - 77, $1 \%$ e 77,4\% respectivamente, em estudo de 404 gestante $^{25}$. Recente trabalho alemão, abrangendo 898 gestantes, também não constatou associação da infecção pelo $H$. pylori com sintomas dispépticos ${ }^{27}$.

No presente estudo, a positividade sorológica ao H. pylori não se associou à presença de fenômenos dispépticos. Das 96 soropositivas, somente $27 \%$ ou $28,1 \%$ manifestaram sintomas dispépticos persistentes. Os sintomas dispépticos da gravidez necessitam ser corretamente diferenciados daqueles da hiperemese gravídica. Esta ocorre em menos de $1 \%$ das gestações, sendo caracterizada por náuseas e vômitos abundantes, que geralmente ocasionam graves distorções do equilíbrio ácidobásico, levando à desidratação, perda de peso e cetonemia ${ }^{25}$.

Mais freqüentemente os estudos constataram significante associação entre a positividade sorológica ao $H$. pylori e esta entidade ${ }^{6,9,28}$, existindo porém trabalhos que não constataram tal associação $0^{8,29}$.

O reconhecimento de subgrupos de gestantes com positividade para $\circ \mathrm{H}$. pylori poderia possibilitar seu tratamento, evitando a transmissão dessas bactérias para o feto; entretanto, a utilização terapêutica desses agentes antimicrobianos durante a gravidez é problemática, não sendo ainda recomendado seu emprego rotineiro?

\section{Conclusão}

Este estudo não associou a presença de infecção pelo $\mathrm{H}$. pyloricom fenômenos dispépticos da gravidez. A única característica materna associada com a presença de infecção pelo $H$. pylori foi a idade. As demais características estudadas nesta pesquisa - índice de massa corpórea, número de partos anteriores e a evolução da gestação - não se associaram à infecção pelo $H$. pylori.

\section{Agradecimentos}

Ao Dr. Sergio Antonio Malaman, Diretor do Serviço de Laboratório Clínico do HSPE-FMO, pelo apoio e facilidades concedidas na execução deste trabalho.

\section{Conflito de interesse: não há}

\section{SUMMARY}

\section{Helicobacter PYLORI INFECTION AND ITS CORRELATION WITH GASTROINTESTINAL SYMPTOMS AND OUTCOME OF PREGNANCY}

OBJECTIVE. The presentstudy intended to investigate whether there was a relationship between pregnant women with dyspeptic complaints and with and without Helycobacter pylori infection in the first and second trimester of pregnancy.

MetHODS. H. pylori seropositivity was determined in 146 (aged 20 to 40 years) antenatal patients at 10 to 23 weeks gestation. H.Pylori ser um Immunoglobulin IgG antibody concentrations were determined by enzyme-linked immunoadsorbent assay (ELISA). Age, obesity, parity, outcome of pregnancy and dyspeptic symptoms were registered in the patients' medical records. Chi-square and Student's t-test were used for statistical analysis of the data.
RESULTS. Prevalence of H. pyloriinfection in the population studied was 65.7\% (96 of 146). H pylori infection was not associated with parity, preterm delivery, obesity or with dyspeptic symptoms. Women positive for H.pylorilgG were older $(30,45 \pm 6.87$ vs $27,96 \pm 7.44, p=0,045565 *)$ than $\mathrm{H}$. pylori negative patients.

CONCLUSION. In this study there was no association of H pyloriinfection with dyspeptic symptoms in pregnancy. nor was its presence associated with obesity, parity and outcome of pregnancy. [Rev Assoc Med Bras 2006; 52(5): 318-22]

KeY wORDS: Helicobacter pylori. Pregnancy. Vomits. Heartburn. Dyspepsia.

\section{REFERÊNCIAS}

I. Dunn BE, Cohen H, Blaser MJ. Helicobacter pylori. Clin Microbiol Rev | 997; | 0:720-4|.

2. McFarlane GA, Munro A. Helicobacter pylori and gastric cancer. Br J Surg 1997;84:1 190-9

3. Versalovic J, Lewandrowski K. Helicobacter pylori Update. Clin Microbiol News 1998; 20: 107-13.

4. Marzio L, Capello G, Ballone E. Evaluation of dyspeptic symptoms in patients with and without Helycobacter pylori infection and normal upper gastrointestinal endoscopy. Dig Liver Dis 2003;35: I 38-42.

5. Salimi-Khayati A, Sharami H, Mansour-Ghanael F, Sadri S, Fallah M. Helicobacter pylori seropositivity and the incidence of hyperemesis gravidarum. Med Sci Monit 2003;9:CR I 2-CR I 5.

6. Frigo P, Lang C, Reisenberger K, Kolbi H, Hirschl AM. Hyperemesis gravidarum associated with Helicobacter pylori seropositivity. Obstet Gynecol 1998;91:615-7.

7. Jacoby EB, Porter KB. Helicobacter pylori infection and persistent hyperemesis gravidarum. Am J Perinatol 1999; 16:85-8.

8. Erdem A, Arslan M, Erdem M, Yildirim G, Himmetoglu O. Detection of Helicobacter Pylori seropositivity in hyperemesis gravidarum and correlation with symptoms. Am J Perinatol 2002; 19:87-92.

9. Koçak I, Akcan Y, Ustun C, Demirel C, Cengiz L, Yamk FF. Helicobacter pylori seropositivity in patients with hyperemesis gravidarum. Intern. J Gynecol Obstet 1999;66:25I - 4.

10. Shirin H, Sadan O, Shevah O, Bruck R, Boaz M, Moss SF, Everon S, Glezerman M, Avni Y. Positive serology for Helicobacter pylori and vomiting in the pregnancy. Arch Gynecol Obstet 2003;22:56-8

I I. Tanriverdi HA, Ustundas Y, Tekin IO, Barut A. Dyspeptic complaints after 20 weeks of gestation are not related to Helicobacter pylori seropositivity. Med Sci Monit 2005; I I:CR 445-8.

12. Eslick GD, Yan P, Xia HH, Murray H, Spurrent B, Talley NJ. Foetal intrauterine growth restrictions with Helicobacter Pylori infection. Aliment Pharmacol Ther 2002; 16:1677-82.

13. Victor AC, Lin AO, Vargas MI, Lopes Filho L, Bromberg SH, Takei K. Avaliação do ELISA IgG no imunodiagnóstico da infecção pelo Helicobacter pylori em pacientes com diagnóstico definitivo, em gestantes assintomáticas e em população aparentemente normal. J Bras Patol 200 I;37: 156.

14. Brown LM. Helicobacter pylori: epidemiology and routes of transmission. Epidemiol Rev 2000;22:283- 97.

15. Kodaira MS, Escobar AMU, Grisi S. Aspectos epidemiológicos do Helicobacter pylori na infância e adolescência. Rev Saúde Pública 2002;36:356- 69 .

16. Rocha GA, Oliveira AMR, Queiroz DMM, Moura SB, Mendes EN. Prevalence of Helicobacter pylori infection in two different populations from Minas Gerais, Brazil. Am J Gastroenterol 1994;88: 13 I3- 7.

17. Souto FJD, Fontes CJF, Rocha GA, Oliveira AMR, Mendes EN, Queiroz DMM. Prevalence of Helicobacter pylori infection in a rural area of the state of Mato Grosso, Brazil. Mem Inst Oswaldo Cruz 1998;93:171-4. 
18. Shmuely H, Obure S, Pássaro DJ, Abuksis G, Yahav J, Fraser G, Pitlik S, et al. Dyspepsia symptoms and Helicobacter pylori infection, Nakuru, Kenya. Emerg Inf. Dis. 2003;9: I 1 03-7.

19. Ashorn M, Miettinen A, Rauska T, Laippala P, Maki M. Seroepidemiological study of Helicobacter pylori infection in infancy. Arch Dis Child Fetal Neonatal I 996;74:FI4I- 2.

20. Kalach N, Desramé J, Bonnet C, Commegeille P, Couturier D, Chaussade $\mathrm{S}$, et al. Helicobacter pylori seroprevalence in asymptomatic pregnant women in France. Clin Diag Lab Immun 2002;9:736- 7.

2I. Blecker U, Lanciers S, Hauser B, Mehta DI, Vandenplas Y. Serology as a valid screening test for Helicobacter pylori infection in asymptomatic subjects. Arch Pathol Lab Med 1995; I 19:30-2.

22. Gold BD, Khanna B, Huang LM, Lee CY, Banatvala N. Helicobacter pylori acquisition in infancy after decline of maternal passive immunity. Pediatr Res 1997;41:64I-6.

23. Bassily S, Frenk RW, Mohareb EW, Wierzba T, Savarino S, Hall E, et al. Seroprevalence of Helicobacter pylori among Egyptian newborns and their mothers:a preliminary report. Am J Trop Med Hyg 1999;61:37-40.

24. Winbery SL, Blaho KE. Dyspepsia in pregnancy. Obstet Gynecol Clin North Am 200 I ; 28:333-50
25. McKenna D, Watson P, Dornan J. Helicobacter pylori infection and dyspepsia in pregnancy. Obstet Gynecol 2003; 1 02:845-9.

26. Reymunde A, Santiago N, Perez L. Helicobacter pylori and severe morning sickness. Am J Gastroenterol 200 I;96:2279-80

27. Weyermann M, Brenner H, Adler G, Yasar Z, Handke-Vesely A, Grab D, et al. Helicobacter pylori infection and the occurrence and severity of gastrointestinal symptoms during pregnancy. Am J Obstet Gynecol 2003; |89:526-3|.

28. Liang-bin X, Jing Y, Ai-bin L, Tang S, Qing-Zhen XIE, Cheng D. Relationship between hyperemesis gravidarum and Helicobacter pylori seropositivity. Chin Med J 2004; I I 7:30 I-2.

29. Wu CY, Tseng JJ, Chou MM, Lin SK, Poon SK, Chen GH. Correlation between Helicobacter pylori infection and gastrointestinal symptoms in pregnancy. Adv Ther 2000; 17:152-8.

Artigo recebido: 18/08/2005

Aceito para publicação: 13/02/2006 